\title{
Regulation of drug resistance and metastasis of gastric cancer cells via the microRNA647-ANK2 axis
}

\author{
WENLONG CAO $^{1 *}$, WEIYUAN WEI $^{1 *}$, ZEXU ZHAN $^{1 *}$, DONGYI XIE $^{1}$, YUBO XIE $^{2}$ and QIANG XIAO ${ }^{1}$ \\ Departments of ${ }^{1}$ Surgery and ${ }^{2}$ Anaesthesiology, The First Affiliated Hospital of Guangxi Medical University, \\ Nanning, Guangxi 530021, P.R. China
}

Received April 18, 2016; Accepted November 22, 2017

DOI: 10.3892/ijmm.2018.3381

\begin{abstract}
Due to a lack of effective methods for early diagnosis, the majority of patients with gastric cancer (GC) are diagnosed during the late stages of the disease, which are often accompanied by metastasis. For these patients, despite being considered an important therapeutic modality in the treatment of cancer, chemotherapy is usually not effective due to multidrug resistance (MDR). The expression levels of $\mathrm{MDR} / \mathrm{metastasis-associated} \mathrm{genes} \mathrm{are} \mathrm{regulated} \mathrm{by} \mathrm{numerous}$ microRNAs (miRNAs/miRs). The expression of miR-647 in GC tissues and SGC7901/VCR cell line (drug resistance to vincristine) was detected by qRT-PCR. The effect of overexpression of miR-647 on drug resistance was evaluated by measuring the half maximal inhibitory concentration $\left(\mathrm{IC}_{50}\right)$ value of SGC-7901/VCR to vincristine and tumor growth in vivo. Moreover, drug-induced cell apoptosis and cell cycle were evaluated by flow cytometry, as well as the ability of cell migration and invasiveness detected by wound healing and transwell assay. Furthermore, underlying targets of miR-647 were predicted by TargetScan and MicroRNA; meanwhile, the expression of ANK2, FAK, MMP2, MMP12,CD44,SNAIL1 were observed by qRT-PCR and western blot analysis. The present study established that the expression levels of miR-647 were downregulated in GC tissues from patients
\end{abstract}

Correspondence to: Professor Qiang Xiao, Department of Surgery, The First Affiliated Hospital of Guangxi Medical University, 6 Shuang-yong Road, Nanning, Guangxi 530021, P.R. China

E-mail: xiaoqiang20050@aliyun.com

Professor Yubo Xie, Department of Anaesthesiology, The First Affiliated Hospital of Guangxi Medical University, 6 Shuang-yong Road, Nanning, Guangxi 530021, P.R. China

E-mail: xieyubo715001@aliyun.com

*Contributed equally

Abbreviation: MDR, multidrug resistance; GC, gastric cancer

Key words: microRNA-647, ankyrin-B, gastric carcinoma, drug resistance, murine model with metastasis and in the vincristine-resistant SGC7901 (SGC-7901/VCR) GC cell line. The $\mathrm{IC}_{50}$ value for vincristine was significantly decreased, whereas the proportion of cells in $G_{0} / G_{1}$ phase and the drug-induced apoptotic rate were significantly increased following upregulation of miR-647. Furthermore, the results demonstrated that miR-647 overexpression led to decreased migration and invasion of SGC-7901/VCR cells. Overexpression of miR-647 was also demonstrated to sensitize tumors to chemotherapy in vivo. In addition, miR-647 overexpression was able to reduce the expression levels of ankyrin-B, focal adhesion kinase, matrix metalloproteinase (MMP)2, MMP12, cluster of differentiation 44 and snail family transcriptional repressor 1 . In conclusion, these findings demonstrated that miR-647 may function as a novel target to ameliorate drug resistance and metastasis of GC cells.

\section{Introduction}

Globally, gastric cancer (GC) has become the second leading cause of cancer-associated mortality in recent centuries (1). Since the majority of patients with GC are diagnosed at the late stages of the disease, which are associated with metastasis, treatment has little effect on survival. For these patients, chemotherapy is the standard treatment; however, patients undergoing chemotherapy often exhibit poor efficacy due to multidrug resistance (MDR), which leads to the failure of chemotherapeutic approaches (2). In general, drug resistance and metastasis/invasion are the two major obstacles to the success of cancer treatment. Furthermore, it has previously been reported that drug-resistant cancer cells possess enhanced invasive ability (3). Despite the fact that numerous mechanisms underlying drug resistance have been extensively investigated, including drug delivery and drug-induced cell apoptosis, the predominant mechanism that induces drug resistance remains largely obscure. Therefore, investigating the mechanisms of regulation that underlie drug resistance-mediated metastasis is the key for the effective diagnosis and treatment of GC.

MicroRNAs (miRNAs/miRs) are noncoding RNAs that consist of 19-24 nucleotides. miRNAs regulate gene expression by targeting the 3'-untranslated regions of mRNAs. Emerging evidence has indicated that miRNAs serve an important role in the drug resistance and metastasis of 
various types of cancer, including GC (4). For example, the miR-200bc/429 cluster sensitizes human cancer cell lines to anticancer drugs by targeting B-cell lymphoma-2, which is a well-known anti-apoptotic gene (5). In addition, our previous experiments revealed that $\mathrm{miR}-1284$ has the potential to reverse drug resistance and inhibit metastasis of GC cells by downregulating eukaryotic translation initiation factor 4A1 (6). Furthermore, Rawlings-Goss et al reported that miR-647 may be associated with the most extensive cancers (breast, testicular, colon, germ cell and gastric cancer) and may be considered a biomarker for GC (7). Meanwhile, our previous study also suggested that miR-647 exerts powerful anti-tumorigenic effects in vitro and in vivo, and may represent a promising therapeutic agent against GC (8). A recent study reported that overexpression of miR-647 results in improved prognosis of Taxol-resistant ovarian cancer cells (9). However, the underlying role of miR-647 in drug resistance and metastasis of GC remains to be elucidated.

The present study aimed to determine the effects of miR-647 on drug resistance and metastasis of GC in vitro and in vivo, and to explore its functional mechanisms.

\section{Materials and methods}

Ethics statement. The present study was approved by the ethical board of the First Affiliated Hospital of Guangxi Medical University (Guangxi, China), and complied with the Declaration of Helsinki. All animal procedures conducted in the present study followed the provisions of the Ethics Committee of the First Affiliated Hospital of Guangxi Medical University. Written informed consent was obtained from all patients.

Human tissue samples and cell lines. Sixteen pairs of GC tissues and adjacent nontumor tissues (located $5 \mathrm{~cm}$ away from the tumor) were collected during surgery at the First Affiliated Hospital of Guangxi Medical University between 2013 and 2015 (age of patients, 47-75 years old; male, 10 cases; female, 6 cases). Tissue samples were preserved in liquid nitrogen. Vincristine-resistant SGC7901 (SGC7901/VCR) cells were obtained from the Cell Bank of the Chinese Academy of Sciences (Shanghai, China). SGC7901/VCR and GES-1 (obtained from the Cell Center of Xiangya Medical University, Changsha, China) cells were cultured in RPMI-1640 (Hyclone; GE Healthcare Life Sciences, Logan, UT, USA), supplemented with $50 \mathrm{mg} / \mathrm{ml}$ penicillin, $100 \mathrm{mg} / \mathrm{ml}$ streptomycin and $10 \%$ fetal bovine serum (Sijiqing Biotech, Co., Ltd, Hangzhou, China). Cells were cultured at $37.8^{\circ} \mathrm{C}$ in an atmosphere containing $5.0 \% \mathrm{CO}_{2}$. Additionally, the groups treated with VCR were supplemented with $0.8 \mu \mathrm{g} / \mathrm{ml}$ vincristine, while the groups treated without VCR were not.

Reverse transcription-quantitative polymerase chain reaction (RT-qPCR). RNA was extracted from SGC7901/VCR cells using TRIzol ${ }^{\circledR}$ (Invitrogen; Thermo Fisher Scientific, Inc., Waltham, MA, USA). According to the manufacturer's protocol, cDNA was reverse transcribed from 1,000 ng RNA using the PrimeScript ${ }^{\mathrm{TM}}$ RT Reagent kit (Takara Bio, Inc., Otsu, Japan). The mRNA and miRNA expression levels were calculated using GAPDH (mRNA) or U6 (miRNA) as a refer- ence gene. RT-qPCR was conducted using SYBR ${ }^{\circledR}$ Premix Ex Taq ${ }^{\mathrm{TM}}$ II (Tli RNaseH Plus) and a ROX Plus Reagent kit (Takara Bio, Inc.) according to the manufacturer's protocol. The thermocycling conditions were as follows: pre-denaturation, $95^{\circ} \mathrm{C}$ for $30 \mathrm{sec}$; amplified reaction, $95^{\circ} \mathrm{C}$ for $5 \mathrm{sec}, 60^{\circ} \mathrm{C}$ for $20 \mathrm{sec}, 40$ cycles; dissociation curve, $95^{\circ} \mathrm{C}$ for $60 \mathrm{sec}$, $55^{\circ} \mathrm{C}$ for $30 \mathrm{sec}, 95^{\circ} \mathrm{C}$ for $30 \mathrm{sec}$. The mRNA and miRNA expression levels were analyzed using the $2^{-\Delta \Delta \mathrm{Cq}}$ method (10). The primer sequences used are listed as Table I.

Antibodies. Rabbit anti-human antibodies specific to focal adhesion kinase (FAK; ab40794), matrix metalloproteinase (MMP)2 (ab92536), MMP12 (ab52897), cluster of differentiation (CD)44 (ab51037), snail family transcriptional repressor 1 (SNAIL1; ab82846) and GAPDH (ab9485); and mouse anti-human antibodies specific to ankyrin-B (ANK2; ab131419) were obtained from Abcam (Cambridge, UK). Infrared-labeled IRDye 800 goat anti-rabbit (P/N 926-32211) and donkey anti-mouse ( $\mathrm{P} / \mathrm{N}$ 926-32212) secondary antibodies were obtained from LI-COR Biosciences (Lincoln, NE, USA).

Bioinformatics analysis. The target genes of miR-647 were analyzed by TargetScan (http://www.targetscan.org) and microRNA.org (http://www.microrna.org/microrna/home. do).

Establishment of stable cell lines. The miR-647 overexpression vector [LV-miR-647-green fluorescent protein (GFP)] and null vector (LV-GFP) were purchased from Shanghai GeneChem Co., Ltd. (Shanghai, China). SGC7901/VCR cells were seeded in 6 -well plates $\left(3 \times 10^{4} /\right.$ well) with antibiotic-free medium. After seeding into 6-well plates for $24 \mathrm{~h}$, cells were infected with the indicated viral supernatants at a multiplicity of infection (MOI) of 100 PFU/cell (MOI=100) with antibiotic-free medium. To obtain stable infection, the cells were incubated in the presence of $700 \mathrm{mg} / \mathrm{ml} \mathrm{G} 418$ (Invitrogen; Thermo Fisher Scientific, Inc.) for $2-3$ weeks at $37^{\circ} \mathrm{C}$ and were then collected. The cells were divided into three groups: The control group, which consisted of untreated SGC7901/VCR cells; the LV-GFP group, which consisted of cells infected with the LV-GFP negative control lentiviral vector; and the LV-miR-647 group, which consisted of cells infected with the LV-miR-647-GFP recombinant lentiviral vector. miR-647 expression in the infected cells was detected by RT-qPCR.

Cytotoxicity assay. Cells $\left(2 \times 10^{3}\right.$ cells/well) were seeded into 96 -well plates. After $24 \mathrm{~h}$, vincristine at six concentrations $(0$, $0.2,0.4,0.8,1.6$ and $3.2 \mathrm{mg} / \mathrm{ml}$ ) was added into the common medium. After $48 \mathrm{~h}\left(37^{\circ} \mathrm{C}\right), 10 \mu \mathrm{l}$ Cell Counting kit- 8 reagent (CCK-8; Dojindo Molecular Technologies, Inc., Kumamoto, Japan) was added and the cells were incubated for $1 \mathrm{~h}$ at $37^{\circ} \mathrm{C}$ in an atmosphere containing $5 \% \mathrm{CO}_{2}$. Subsequently, absorbance was detected at a wavelength of $450 \mathrm{~nm}$. The half maximal inhibitory concentration $\left(\mathrm{IC}_{50}\right)$ was calculated according to the relative survival curve. Each experiment was processed in quadruplicate.

Cell cycle analysis. Cells were washed twice with PBS and fixed with $70 \%$ ethanol for $12 \mathrm{~h}$ at $4^{\circ} \mathrm{C}$. The cells were 
Table I. Primer sequences for reverse transcription-quantitative polymerase chain reaction.

\begin{tabular}{ll}
\hline Gene & \multicolumn{1}{c}{ Primer sequences } \\
\hline miR-647 & F: 5'-GUGGCUGCACUCACUUCCUUC-3' \\
& R:5'-CTCAACTGGTGTCGTGGA-3' \\
U6 & F: 5'-TTATGGGTCCTAGCCTGAC-3' \\
& R:5'-CACTATTGCGGGTCTGC-3' \\
ANK2 & F: 5'-CAGCTACATTGTGTGGCATTCTA-3' \\
& R:5'-CTACAGTGCAGTGGCCAGAAG-3' \\
FAK & F: 5'-CAACCACCTGGGCCAGTATTATC-3' \\
& R:5'-CCATAGCAGGCCACATGCTTTA-3' \\
MMP2 & F: 5'-CCGTGTTTGCCATCTGTTTAG-3' \\
& R:5'-AGGTTCTCTTGCTGTTTACTTTGGA-3' \\
MMP12 & F: 5'-ACGTGGCATTCAGTCCCTGT-3' \\
& R:5'-AACACTGGTCTTTGGTCTCTCAGAA-3' \\
CD44 & F: 5'-CAGGGCTGGGCTTAGACAGA-3' \\
& R:5'-CTGGCCAATGATGTTCACAGA-3' \\
SNAIL1 & F:5'-CAGACCCACTCAGATGTCAAGAA-3' \\
& R:5'-GGGCAGGTATGGAGAGGAAGA-3' \\
GAPDH & F: 5'-GCACCGTCAAGGCTGAGAAC-3' \\
& R:5'-TGGTGAAGACGCCAGTGGA-3'
\end{tabular}

ANK2, ankyrin-B; CD44, cluster of differentiation; F, forward; FAK, focal adhesion kinase; MMP, matrix metalloproteinase; R, reverse; SNAIL1, snail family transcriptional repressor 1 .

then incubated in a solution containing RNase $(200 \mathrm{ng} / \mathrm{ml}$; RT405-12; Tiangen, Beijing, China) and propidium iodide $(0.05 \mathrm{mg} / \mathrm{ml})$ at room temperature for $30 \mathrm{~min}$. Results were then analyzed by flow cytometry (BD Biosciences, San Jose, CA, USA). The data was analyzed with the CellQuest ${ }^{\mathrm{TM}}$ software (BD Biosciences).

Apoptosis assay. Apoptosis was determined by flow cytometry using the Apoptosis Detection kit (BD Biosciences), according to the manufacturer's protocol.Briefly, the cells were incubated in a solution containing Annexin V-phycoerythrin $(5 \mu \mathrm{l} / \mathrm{ml})$ and 7 -amino-actinomycin $\mathrm{D}(5 \mu \mathrm{l} / \mathrm{ml})$ at $4^{\circ} \mathrm{C}$ in the dark for $30 \mathrm{~min}$. Results were then analyzed by flow cytometry. The data was analyzed with the CellQuest ${ }^{\mathrm{TM}}$ software (BD Biosciences).

Wound healing assay. In order to inhibit cell proliferation, cells were cultured with mitomycin C $(10 \mu \mathrm{g} / \mathrm{ml}$;

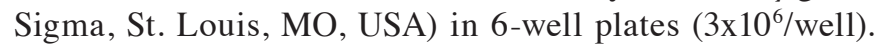
Subsequently, a straight wound was generated using a $200 \mu 1$ sterilized pipet tip and the cells were washed twice with PBS to remove nonadherent cells. Wound healing was observed at 0, 24, 48 and $72 \mathrm{~h}$ under a microscope (Nikon TS100; Nikon, Tokyo, Japan), and relative motility was calculated according to the following formula: Relative motility = (initial distance-a time-point distance)/initial distance x $100 \%$. A time-point distance could be 24, 48, 72 h (Fig. 2C).
Cell invasion assay. Serum-free RPMI-1640 (75 $\mu \mathrm{l})$ containing $1 \mu \mathrm{g} / \mathrm{ml}$ Matrigel (BD Biosciences) was added to the upper chamber of Transwell apparatus $(6.5 \mathrm{~mm}$; Corning Inc., Corning, NY, USA). Cells $\left(5 \times 10^{4}\right)$ were seeded into the upper chamber alongside serum-free $200 \mu \mathrm{l}$ RPMI-1640, whereas $700 \mu \mathrm{l}$ RPMI-1640 containing 5\% fetal bovine serum was added to the lower chamber. After $24 \mathrm{~h}\left(37^{\circ} \mathrm{C}\right)$, the cells that had invaded through the membranes were stained with Giemsa, and the number of visible cells was counted in six random views at $\mathrm{x} 200$ magnification using a microscope (Nikon TS100; Nikon).

Western blot analysis. Proteins were extracted from cells using cell lysis buffer (Beijing Solarbio Science \& Technology Co., Ltd., Beijing, China). Subsequently, proteins were separated by $10 \%$ SDS-PAGE and were transferred onto nitrocellulose membranes. Protein concentration was measured by BCA Protein Assay kit (Beyotime Institute of Biotechnology, Shanghai, China). The protein samples $(100 \mu \mathrm{g})$ were loaded onto the gel. The membranes were incubated in blocking buffer for $1 \mathrm{~h}$ at room temperature. The membranes were then incubated with primary antibodies $(1: 1,000)$ overnight at $4{ }^{\circ} \mathrm{C}$ and were washed with Tris-buffered saline containing $0.1 \%$ Tween. The membranes were then incubated with infrared-labeled secondary antibodies $(1: 10,000)$ for $1 \mathrm{~h}$ at room temperature and LI-COR Odyssey Imaging System (LI-COR Biosciences) was used to analyze densitometry. GAPDH $(1: 1,000)$ was used as an internal control.

Effects of miR-647 on GC cells in vivo. BALBC/c nude mice (age, 4-5 weeks) were provided by the Guangxi Animal Center (Nanning, China). All animal experimental studies were implemented in the Guangxi Animal Center. Mice were maintained in specific-pathogen-free surroundings, and feeding schedules conformed to the guidelines of the Ethics Committee of Guangxi Medical University (temperature, $20-25^{\circ} \mathrm{C}$; humidity, 40-70\%; light and dark $12 \mathrm{~h}$ respectively; having access to sterilized food and water). All the mice lived under these conditions since they were born. Tumors were implanted by an injection of $4 \times 10^{7} \mathrm{SGC}-7901 / \mathrm{VCR}$ cells suspended in $100 \mu \mathrm{l} \mathrm{PBS}$ (Beyotime Institute of Biotechnology) into the armpit region of nude mice. After 4 days, $5 \mathrm{~mm}$-diameter tumors were achieved. The mice were randomly divided into six groups (n=6 mice/group): Control-nonVCR group, LV-GFP-nonVCR group, LV-miR-647-nonVCR group, control group, LV-GFP group and LV-miR-647 group. An intratumoral injection of LV-miR-647-GFP or LV-GFP (5x10 6 TU) in $100 \mu 1$ PBS was directed into the tumors of LV-miR-647-nonVCR and LV-miR-647 groups, or LV-GFP-nonVCR and LV-GFP groups, respectively; a similar volume of PBS was injected into the control-nonVCR and control groups. VCR was additionally administered by intraperitoneal injection $(200 \mathrm{ng} / \mathrm{kg})$ into the control, LV-GFP and LV-miR-647 groups. Following the first operation (first intratumoral injection), the mice received the same treatment every 2 days. Tumor size was calculated every 4 days using a vernier caliper. The length (a) and the width (b) were measured, and tumor volume was determined as follows: Tumor volume $=a \mathrm{xb}^{2} / 2$. Relative tumor volume (RTV) was estimated according to the following equation: $\mathrm{RTV}=\mathrm{V}_{\mathrm{t}} / \mathrm{V}_{0}$ 

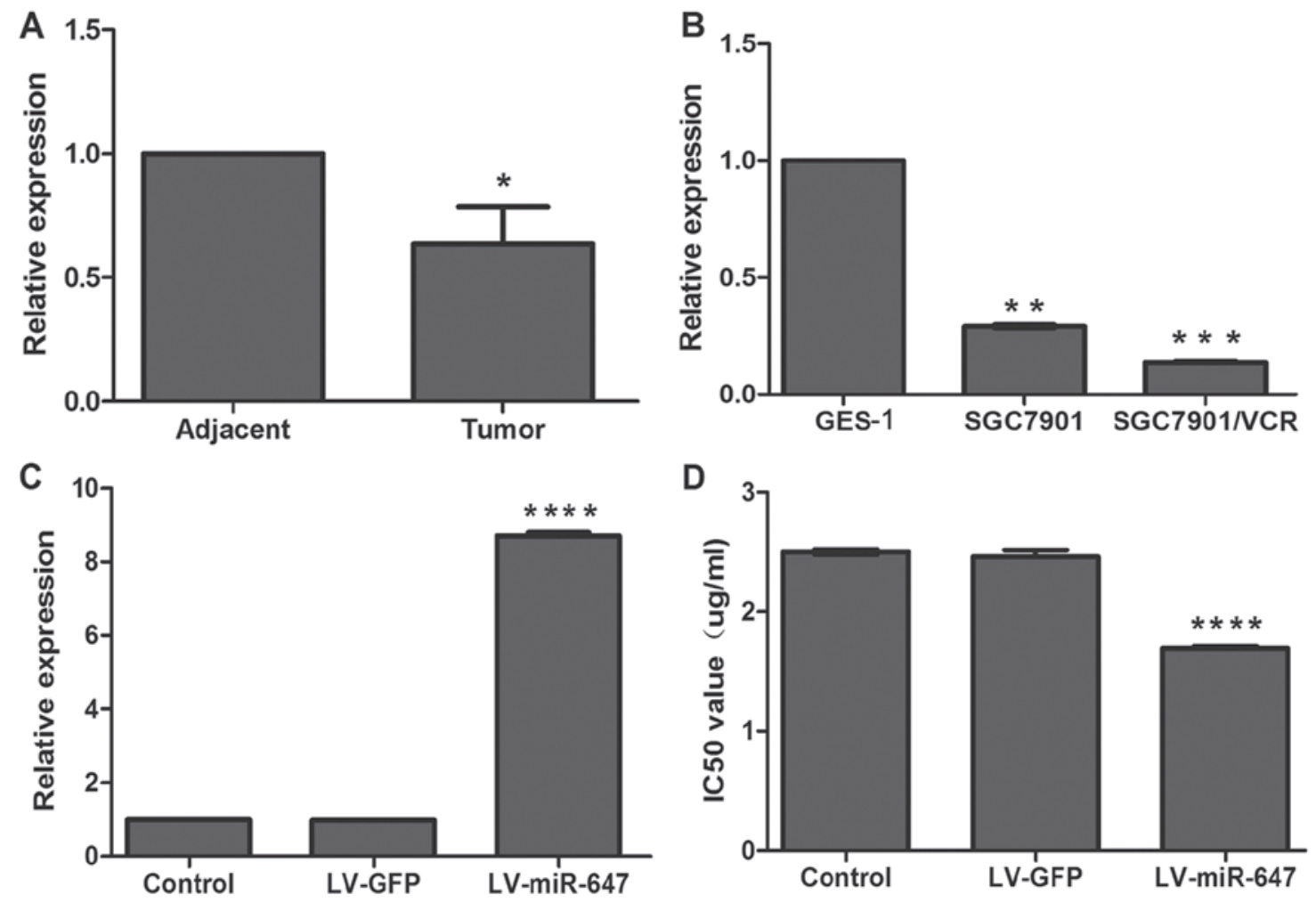

Figure 1. miR-647 expression is decreased in GC tissue specimens and drug-resistant GC cells, and can overcome drug resistance in GC cells. (A) miR-647 expression in GC tissue specimens from patients with distant metastasis and adjacent nontumor tissues was detected by RT-qPCR (n=4). " $\mathrm{P}<0.05$ vs. adjacent nontumor tissues. (B) miR-647 expression in SGC7901/VCR, SGC7901 and GES-1 cells was detected by RT-qPCR ( $\mathrm{n}=4)$. ${ }^{* *} \mathrm{P}<0.05$ vs. GES-1 cells; ${ }^{* * *} \mathrm{P}<0.05$ vs. SGC7901 and GES-1 cells. (C) miR-647 expression was analyzed by RT-qPCR following infection ( $\mathrm{n}=4)$. (D) $\mathrm{IC}_{50}$ values for vincristine were calculated (n=4). ${ }_{* * * *} \mathrm{P}<0.05$ vs. LV-GFP and control groups. Data are presented as the means \pm standard error of the mean. GC, gastric cancer; GFP, green fluorescent protein; $\mathrm{IC}_{50}$, half maximal inhibitory concentration; miR-647, microRNA-647; RT-qPCR, reverse transcription-quantitative polymerase chain reaction.

where $\mathrm{V}_{0}$ refers to the tumor volume at the time of intraperitoneal injection; and $\mathrm{V}_{\mathrm{t}}$ refers to the tumor volume at the next measurement. After 20 days of feeding, the mice were sacrificed and tumors were evaluated. Tumors were immersed in $4 \%$ formaldehyde, and were embedded in paraffin following dehydration with an ethanol series. The tumors were fixated at room temperature for $24 \mathrm{~h}$. Subsequently, tumor segments were dewaxed, rehydrated and stained with hematoxylin and eosin (H\&E). Sections were then observed under a middle power field (x200) (Nikon TS100; Nikon).

Statistical analysis. Data are presented as the mean \pm standard error of the mean. Statistical analyses were conducted using SPSS 13.0 (SPSS, Inc., Chicago, IL, USA). Data were analyzed using Student's t-test, one-way analysis of variance (ANOVA) or $\chi^{2}$ test. The results between 3 groups were compared by ANOVA and the results between 2 groups were compared by t-test. $\mathrm{P}<0.05$ was considered to indicate a statistically significant difference.

\section{Results}

miR-647 is decreased in GC tissue specimens and drug-resistant GC cells. To ascertain whether miR-647 was associated with the generation of GC metastasis and drug resistance, the present study detected miR-647 expression in GC tissue specimens from patients with distant metastasis and compared them to adjacent nontumor tissues. In 11 out of 16 GC cases,
miR-647 expression was downregulated compared with in the adjacent nontumor tissues, as determined by RT-qPCR $(\mathrm{P}<0.05$; Fig. 1A). Furthermore, miR-647 expression was detected in SGC7901/VCR and SGC7901 cells, and in the human gastric epithelial cell line GES-1. The expression levels of miR-647 in GES-1 cells were 3.4- and 7.3-fold higher compared with in SGC7901 and SGC7901/VCR cells, respectively $(\mathrm{P}<0.05)$; and miR-647 expression was 2.1-fold higher in SGC7901 cells compared with in SGC7901/VCR cells ( $\mathrm{P}<0.05$; Fig. 1B). These results indicated that miR-647 may be associated with distant metastasis of GC and drug-resistant GC cells.

miR-647 recombinant lentiviral vectors increase miR-647 expression. To test the hypothesis that miR-647 recombinant lentiviral vectors could increase the expression levels of miR-647 in SGC7901/VCR cells, these cells were infected with LV-miR-647-GFP (LV-miR-647 group) and LV-GFP (LV-GFP group). The expression levels of miR-647 in cells from the LV-miR-647 group were 8.7- and 8.3-fold higher compared with in the LV-GFP and control groups, respectively $(\mathrm{P}<0.05$; Fig. 1C). These observations suggested that LV-miR-647-GFP may upregulate miR-647 expression in SGC7901/VCR cells.

miR-647 is able to overcome GC drug resistance in vitro. SGC7901/VCR cells were selected from SGC7901 cells using the anticancer drug vincristine, and the expression levels of miR-647 were observed to be downregulated in SGC7901/VCR cells compared with in SGC7901 and GES-1 
A
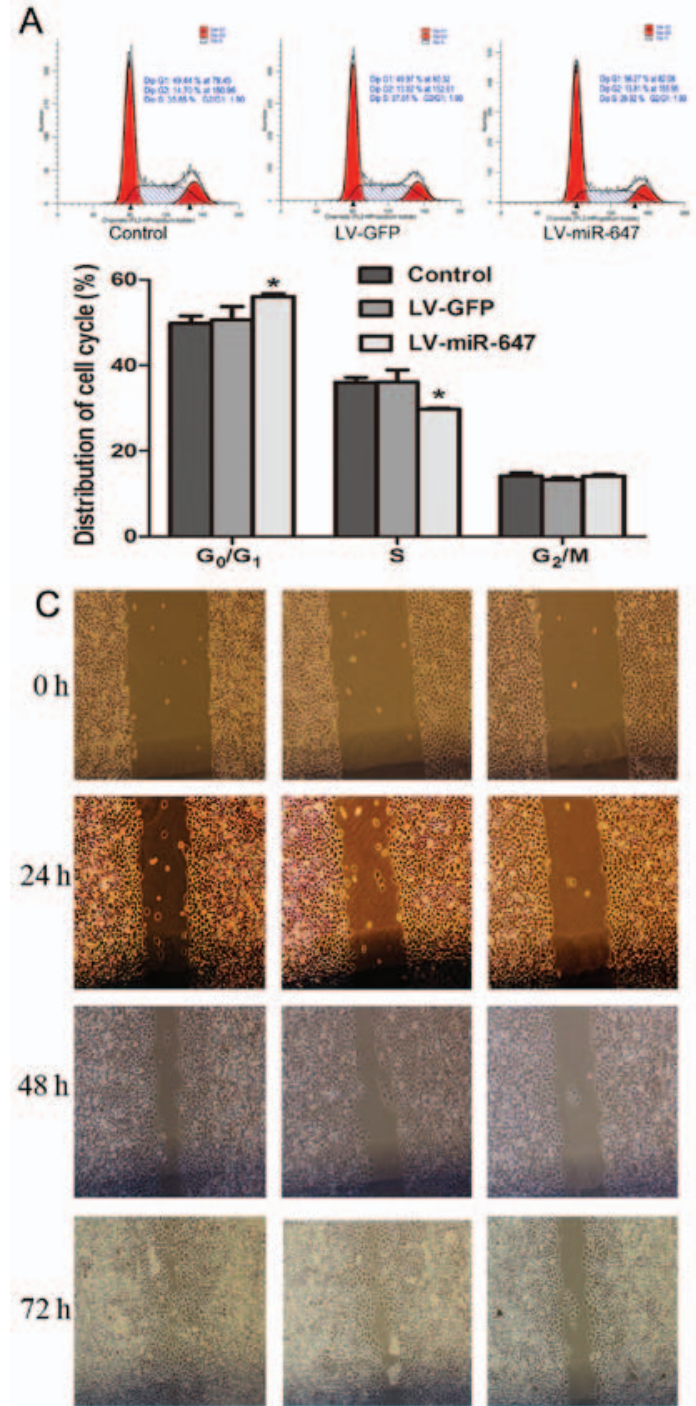

Control

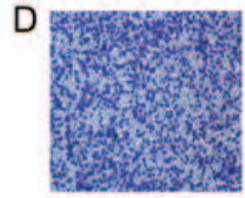

Control
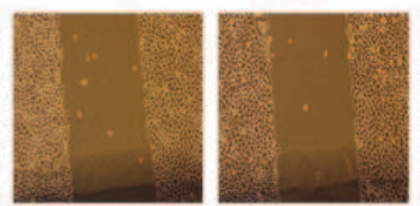
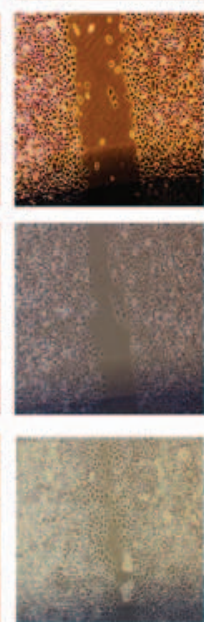

LV-GFP

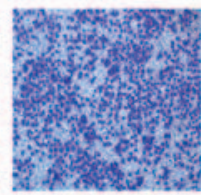

LV-GFP
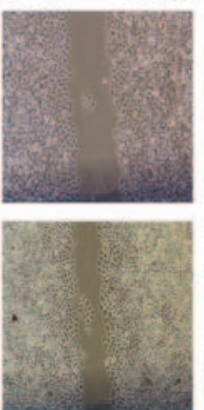

LV-miR-647

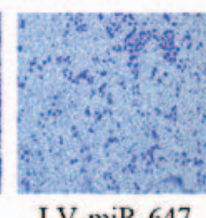

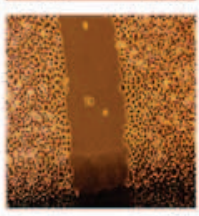

B
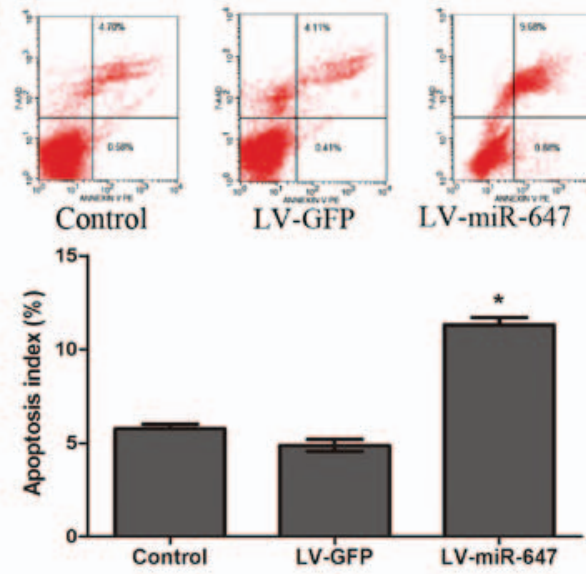
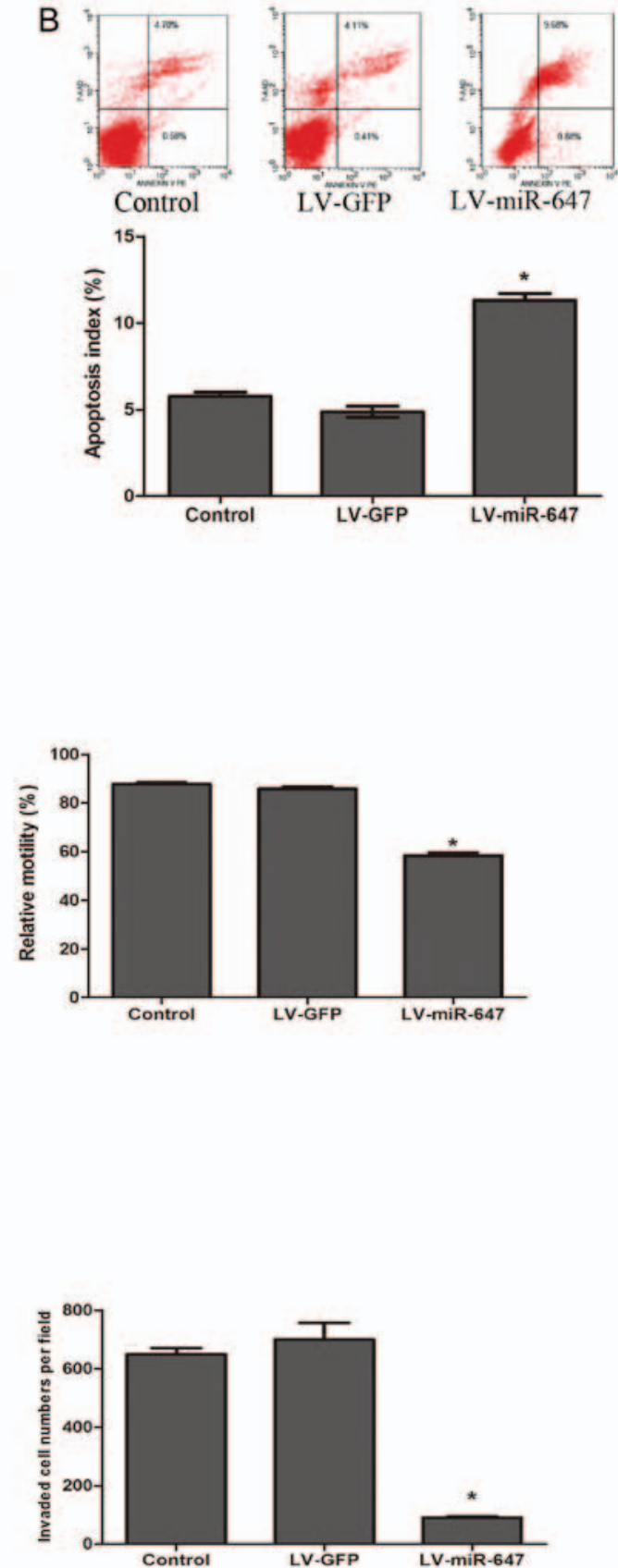

Figure 2. miR-647 prevents cells from entering S phase, accelerates drug-induced apoptosis, and decreases migration and invasion of GC cells. (A) Cell cycle progression of SGC7901/VCR cells following miR-647 infection and vincristine treatment ( $24 \mathrm{~h}$; concentration, $0.8 \mu \mathrm{g} / \mathrm{ml})$ was determined by flow cytometry (n=4). (B) Percentages of apoptotic SGC7901/VCR cells following miR-647 infection and vincristine treatment $(24 \mathrm{~h}$; concentration, $0.8 \mu \mathrm{g} / \mathrm{ml})$ was determined by flow cytometry $(\mathrm{n}=4)$. (C) Migratory ability of SGC7901/VCR cells following miR-647 infection was investigated by wound healing assay (magnification, $\mathrm{x} 100)(\mathrm{n}=3)$. (D) Invasion of SGC7901/VCR cells following miR-647 infection was investigated by Transwell assay (magnification, $\mathrm{x} 200$ ) $(n=3)$. "P<0.05 vs. the LV-GFP and control groups. Data are presented as the means \pm standard error of the mean. GC, gastric cancer; GFP, green fluorescent protein; miR-647, microRNA-647.

cells (Fig. 1B). The present study hypothesized that miR-647 could regulate drug resistance in GC cells; therefore, the effects of miR-647 overexpression on the drug sensitivity of SGC7901/VCR cells was determined using a CCK-8 assay. The results demonstrated that the $\mathrm{IC}_{50}$ value for vincristine in the LV-miR-647 group was $1.69 \pm 0.03 \mu \mathrm{g} / \mathrm{ml}$, which was significantly lower than that in the LV-GFP $(2.46 \pm 0.10 \mu \mathrm{g} / \mathrm{ml})$ and control groups $(2.50 \pm 0.02 \mu \mathrm{g} / \mathrm{ml})(\mathrm{P}<0.05$; Fig. 1D). These findings indicated that miR-647 may markedly enhance the sensitivity of GC to vincristine.
miR-647 promotes cell cycle arrest at the $G_{0} / G_{l}$ phase. To explore whether miR-647 overexpression-induced drug sensitivity was mediated by an alteration in a specific phase of the cell cycle, flow cytometry was used to detect the number of cells in each phase of the cell cycle in each group. The results indicated that the number of cells in $\mathrm{G}_{0} / \mathrm{G}_{1}$ phase was markedly increased, whereas the number of cells in $\mathrm{S}$ phase was decreased in the LV-miR-647 group $(\mathrm{P}<0.05$; Fig. 2A). There was no statistical significance in the groups without vincristine treatment (data not shown). These findings 

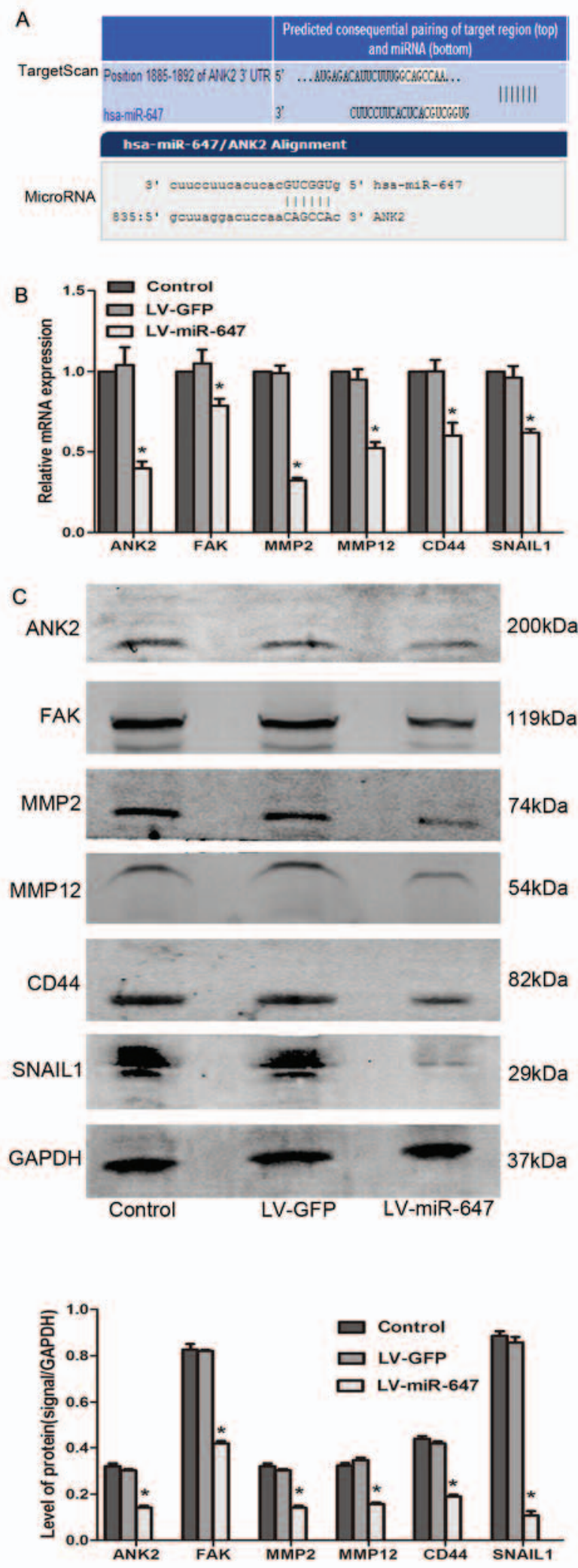

Figure 3. miR-647 modulates drug resistance by reducing ANK2, FAK, MMP2, MMP12, CD44 and SNAIL1 expression. (A) TargetScan and microRNA.org were used to predict the target gene of miR-647. (B) ANK2, FAK, MMP2, MMP12, CD44 and SNAIL1 mRNA expression was detected by reverse transcription-quantitative polymerase chain reaction $(n=4)$. (C) ANK2, FAK, MMP2, MMP12, CD44 and SNAIL1 protein expression was detected using western blotting $(n=3)$. "P $<0.05$ vs. the $L V-G F P$ and control groups. Data are presented as the means \pm standard error of the mean. ANK2, ankyrin-B; CD44, cluster of differentiation 44; FAK, focal adhesion kinase; GFP, green fluorescent protein; miR-647, microRNA-647; MMP, matrix metalloproteinase; SNAIL1, snail family transcriptional repressor 1 . indicated that reversal of drug resistance is associated with miR-647, which may prevent cells from entering $\mathrm{S}$ phase and leads to a reduction in the number of cells.

miR-647 accelerates drug-induced apoptosis. Enhancing drug-induced apoptosis is a key mechanism for reversing drug resistance. The present results verified that following incubation with vincristine, the apoptotic rate was markedly increased in the LV-miR-647 group compared with in the LV-GFP and control groups $(\mathrm{P}<0.05$; Fig. $2 \mathrm{~B})$. There was no statistical significance in the groups without vincristine treatment (data not shown). These results indicated that miR-647 increased drug-induced apoptosis of GC cells, thereby reversing drug resistance.

miR-647 decreases migration and invasion of GC cells. Since miR-647 was downregulated in tissues from patients with GC and distant metastasis, it was hypothesized that miR-647 may be correlated with the migration and invasion of cells. To test this hypothesis, the migratory and invasive ability of each group was determined using wound healing and Transwell assays. After $72 \mathrm{~h}$, the LV-miR-647 group exhibited reduced migratory ability compared with in the LV-GFP and control groups. The relative motility rates of cells in the LV-miR-647, LV-GFP and control groups were $58.43 \pm 2.08,85.79 \pm 0.96$ and $87.77 \pm 1.06 \%$, respectively $(\mathrm{P}<0.05$; Fig. $2 \mathrm{C})$. With regards to cell invasion, the Transwell assay indicated that the number of cells that invaded through the Matrigel membrane in the LV-miR-647 group was reduced compared with in the LV-GFP and control groups; the number of cells in the LV-GFP and control groups that invaded through the membrane was 7.7and 7.1-fold greater compared with in the LV-miR-647 group, respectively $(\mathrm{P}<0.05$; Fig. 2D). These results suggested that miR-647 may decrease the migration and invasion of GC cells.

miR-647 modulates drug resistance by reducing the expression levels of ANK2, FAK, MMP2, MMP12, CD44 and SNAIL2. The data generated using TargetScan 6.2 and microRNA.org databases revealed that ANK2 is an underlying target of miR-647 (Fig. 3A). In the present study, the mRNA and protein expression levels of ANK2, FAK, MMP2, MMP12, CD44 and SNAIL1 were significantly reduced in the LV-miR-647 group compared with in the LV-GFP and control groups $(\mathrm{P}<0.05$; Fig. $3 \mathrm{~B}$ and $\mathrm{C})$, thus providing a mechanistic basis for the reversal of drug resistance in drug-resistant GC cells upon miR-647 overexpression.

miR-647 reverses drug resistance in GC in vivo. To further support the in vitro observations in the present study, the effects of miR-647 were determined on the growth of SGC7901/VCR cells in vivo; LV-miR-647-GFP and LV-GFP were intratumorally injected into SGC7901/VCR tumors that were transplanted subcutaneously into nude mice. A total of 20 days after intratumoral injection, the RTV of the LV-miR-647 group was 2.04 \pm 0.10 , which was significantly reduced compared with in the LV-GFP group $(2.94 \pm 0.40)$ and the control group $(2.82 \pm 0.46) \quad(\mathrm{P}<0.05$; Fig. 4A). However, there was no statistical significance among the Control-nonVCR, LV-GFP-nonVCR and LV-miR-647-nonVCR groups. RT-qPCR confirmed that 
A

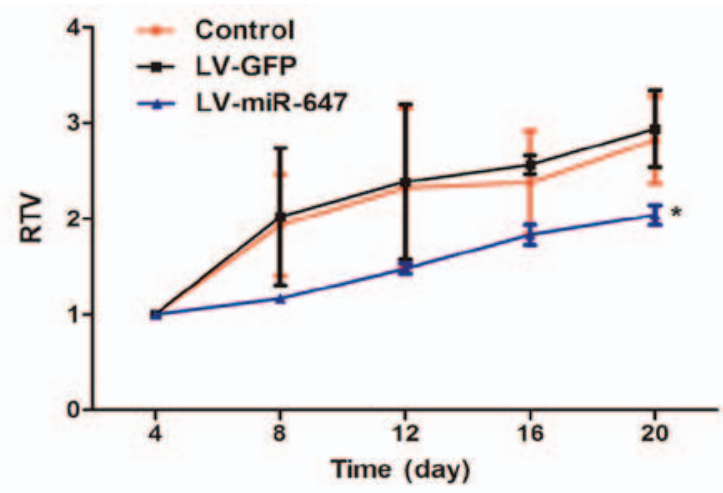

B

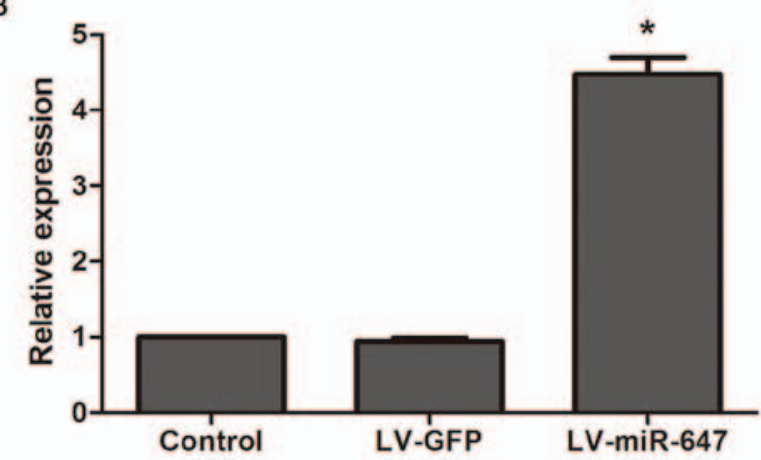

C

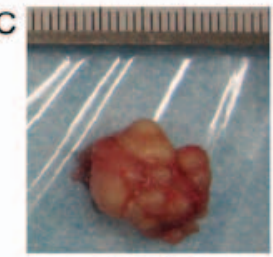

Control

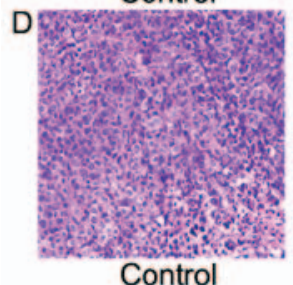

Control

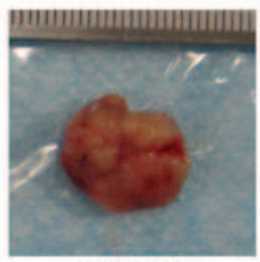

LV-GFP

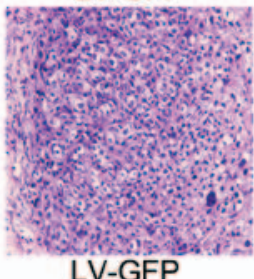

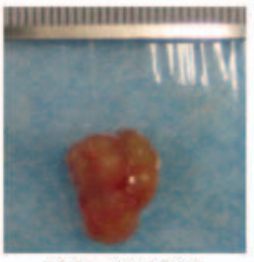

LV-miR-647

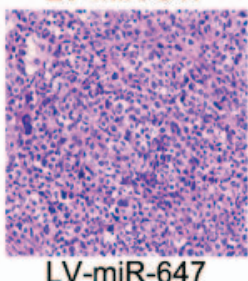

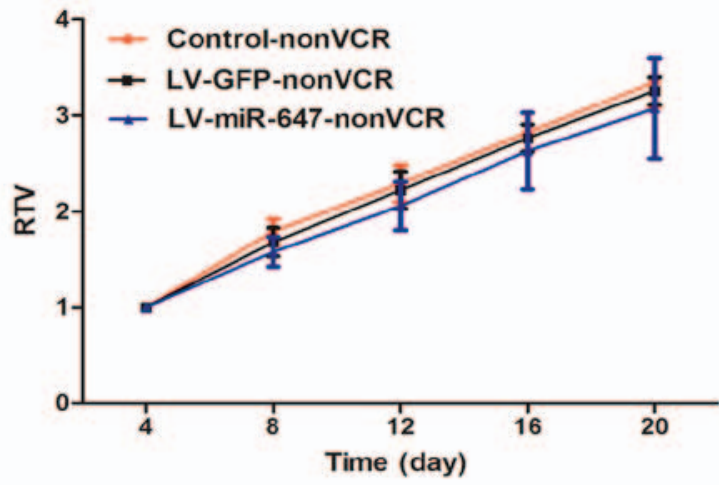
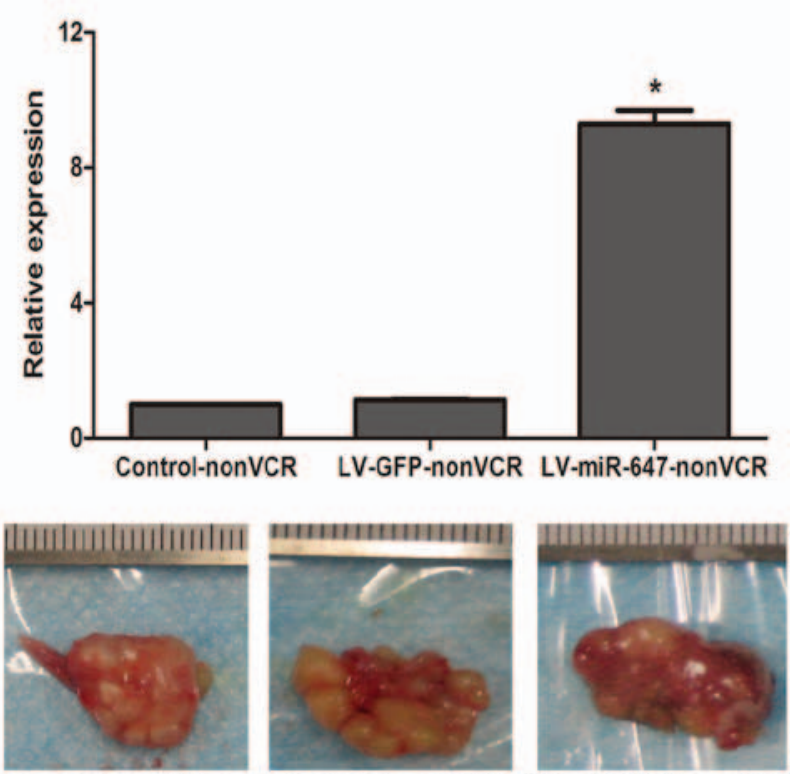

Control-nonVCR
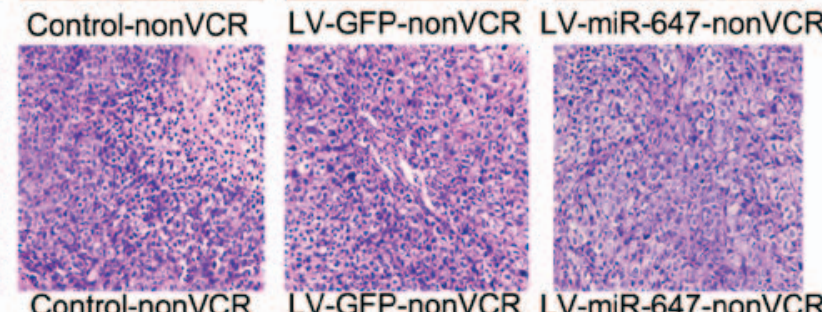

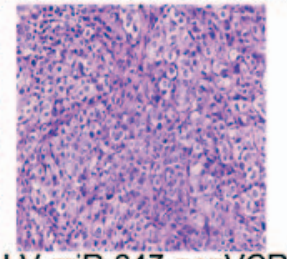

Control-nonVCR

Figure 4. miR-647 reverses drug resistance in gastric cancer in vivo. (A) RTV of each group (n=6). (B) miR-647 expression in each group, as determined by reverse transcription-quantitative polymerase chain reaction $(n=4)$. (C) Xenografts from each group. (D) Tumor segments stained by hematoxylin and eosin (magnification, $\mathrm{x} 200$ ). ${ }^{*} \mathrm{P}<0.05$ vs. LV-GFP or LV-GFP-nonVCR groups and control or control-nonVCR groups. Data are presented as the means \pm standard error of the mean. GFP, green fluorescent protein; miR-647, microRNA-647; RTV, relative tumor volume.

miR-647 expression was increased in xenografts from the LV-miR-647 or LV-miR-647-nonVCR groups compared with in the LV-GFP or LV-GFP-nonVCR groups and the control or control-nonVCR groups, respectively $(\mathrm{P}<0.05$; Fig. 4B). Images of the xenografts from each group are presented in Fig. 4C, and were confirmed by H\&E staining analysis (Fig. 4D). H\&E staining confirmed that the lumps were transplantation tumors. These data suggested that miR-647 may reverse drug resistance in GC in vivo.

\section{Discussion}

Due to the absence of efficacious methods for early diagnosis and management of tumor metastases, the majority of patients with GC are often unable to be treated by surgery.
Chemotherapy is considered to be the preferable therapeutic option for these patients; however, the occurrence of MDR has become a vital factor associated with the failure of routine chemotherapy, resulting in metastasis of the most malignant tumors (11). Drug resistance and tumor metastasis/invasion have been identified as the classical hallmarks of cancer malignancy, and are major causes of poor clinical outcome in patients with cancer (12-15). Therefore, discovery of novel molecular biomarkers and targets for the diagnosis and treatment of GC is imperative.

Accumulating evidence has suggested that miRNAs are involved in the initial development of drug resistance and metastasis in patients with GC (16). Our previous study demonstrated that miR-1284 functions as a novel regulator to reduce drug resistance and metastasis of GC (6). Notably, 
Rawlings-Goss et al (7) reported that miR-647 is associated with malignant cancer phenotypes and is often used as a biomarker for GC. Furthermore, dysregulation of miR-647 has been reported to be associated with Taxol resistance in ovarian cancer (9). However, the detailed association between miR-647 and GC tumorigenesis remains unclear. The mechanism underlying the involvement of miR-647 in drug resistance and metastasis of GC has yet to be elucidated.

The present study indicated that the expression levels of miR-647 in GC tissues and SGC7901/VCR cells were reduced compared with in the controls. Overexpression of miR-647 reversed vincristine resistance in SGC7901/VCR cells, prevented cells from entering $\mathrm{S}$ phase of the cell cycle and induced cell apoptosis. Furthermore, overexpression of miR-647 downregulated migration and invasion of SGC7901/VCR cells, and sensitized tumors to chemotherapy in vivo, as demonstrated by a decrease in RTV in nude mice. To the best of our knowledge, the present data is the first to demonstrate that miR-647 may function as a novel regulator of MDR in GC.

The exact mechanism underlying the functions of miR-647 in GC MDR remains unclear. The present study provided evidence to suggest that miR-647 overexpression regulates SGC7901/VCR cell drug resistance by reducing ANK2, FAK, MMP2, MMP12, CD44 and SNAIL1 expression. Previous studies have reported that ANK2 encodes one of three ankyrins: ankyrin-R (ANK1), ankyrin-B (ANK2) and ankyrin-G (ANK3), which belong to a cytoskeletal protein family $(17,18)$. This particular family is known to be associated with various integral membrane proteins $(17,18)$. Overexpression of ankyrins has been observed in various cancer cells, including prostate, breast, and ovarian cancers (19-21), and they are known to serve crucial roles in cell growth, membrane transportation, metastasis and migration of cancer cells $(18,19)$. The present study demonstrated that miR-647 can regulate ANK2 activity and suppress ANK2 expression, thus indicating that miR-647 may produce biological functions via downregulating ANK2. In addition, a previous study reported that ANK2 is overexpressed in pancreatic tumors, whereas downregulation of ANK2 attenuated growth and invasion of pancreatic cancer (22). In addition, Savas et al revealed that drug resistance in cancer cells is associated with ANK2 (23). However, there is currently insufficient evidence to confirm the exact role of ANK2 in drug resistance and metastasis of GC. To the best of our knowledge, the present findings that miR-647 reverses drug resistance in GC by regulating ANK 2 are the first to provide evidence regarding the relationship between miR-647 and ANK2, and their detailed function in drug resistance and metastasis.

Ankyrins, including ANK1, ANK2 and ANK3, which link various transmembrane proteins to the actin network, also bind domains in CD44 (24). Furthermore, inhibiting CD44 may decrease the expression of SNAIL1 and the invasive ability of pancreatic cancer cells (25). These previous findings were consistent with those of the present study, which indicated that overexpression of miR-647 reversed drug resistance, decreased invasion of SGC7901/VCR cells, and attenuated ANK2, CD44 and SNAIL1 activation. CD44 is a member of the hyaluronan receptor family, which has been reported to be associated with drug resistance (26). Furthermore, there is a strong evidence to suggest that small interfering RNA against CD44 may reduce drug resistance through a decrease in transport efficacy, which can in turn enhance cytoplasmic drug concentration (27). In addition, in head and neck squamous cell carcinoma, the deletion of SNAIL1 has been reported to contribute to the inhibition of migration and invasion, and MDR reversal, which further supports the present findings (28). In the present study, miR-647 expression was revealed to decrease the expression of ANK2/CD44/SNAIL1 signaling pathways, directly or indirectly, which may be responsible for overcoming drug resistance in GC cells in vitro and in vivo.

Previous studies have reported that ANK2 may have an effect on signal transduction mediated by FAK activity. Furthermore, FAK activity has a pivotal role in the secretion of MMPs $(22,29)$. In the present study, miR-647 overexpression reversed drug resistance and inhibited metastasis of SGC7901/VCR cells (invasion/migration of the cells), which was accompanied by reduced ANK2 activation and decreased FAK, MMP2 and MMP12 expression. The present study indicated that the miR-647/ANK2/FAK/MMP2/MMP12 signaling pathway is an uncommon integrated network that may mediate the metastasis of GC. As a potential predictor for tumor metastasis, decreased FAK is involved in the inhibition of tumor metastasis and invasion in GC (30). In addition, MMP2 and MMP12 belong to the MMP family, which are well known for their essential roles in tumor metastasis and invasiveness $(31,32)$. Previous studies have also reported that inhibiting MMP2 and MMP12 suppresses the invasion of gastric and lung cancers $(33,34)$. Notably, primary tumors and metastasis exhibit varying levels of drug resistance; metastasis is generally associated with more severe drug-resistance (35). Therefore, inhibiting metastasis may provide a key strategy to prevent drug resistance.

In conclusion, the present study is the first, to the best of our knowledge, to provide information regarding the association between miR-647 and ANK2 in the drug resistance, thus indicating the potential role of miR-647 in the regulation of drug resistance and metastasis. Therefore, miR-647 may be considered a biomarker of GC, and more studies are required to confirm that targeting this gene may aid in the diagnosis and treatment of GC.

\section{Acknowledgements}

The present study was supported by grants from the National Natural Science Foundation of China (grant nos. 81660511, 30860273 and 81060201), the Natural Science Foundation of Guangxi (grant no. 2015GXNSFDA227001), the Key Health Science Foundation of Guangxi (grant no. 14124004-1-9), and the Innovation Project of Guangxi Graduate Education.

\section{Competing interests}

The authors declare there is no competing interest.

\section{References}

1. Jemal A, Bray F, Center MM, Ferlay J, Ward E and Forman D: Global cancer statistics. CA Cancer J Clin 61: 69-90, 2011.

2. Fan D, Zhang X, Chen X, Mou Z, Hu J, Zhou S, Ding J and Wu K: Bird's-eye view on gastric cancer research of the past 25 years. J Gastroenterol Hepatol 20: 360-365, 2005. 
3. Lu L, Zhou D, Jiang X, Song K, Li K and Ding W: Loss of E-cadherin in multidrug resistant breast cancer cell line MCF-7/Adr: Possible implication in the enhanced invasive ability. Eur Rev Med Pharmacol Sci 16: 1271-1279, 2012.

4. Chen CZ: MicroRNAs as oncogenes and tumor suppressors. N Engl J Med 353: 1768-1771, 2005.

5. Zhu W, Xu H, Zhu D, Zhi H, Wang T, Wang J, Jiang B, Shu Y and Liu P: miR-200bc/429 cluster modulates multidrug resistance of human cancer cell lines by targeting BCL2 and XIAP. Cancer Chemother Pharmacol 69: 723-731, 2012.

6. Cao W, Wei W, Zhan Z, Xie Y and Xiao Q: miR-1284 modulates multidrug resistance of gastric cancer cells by targeting EIF4A1. Oncol Rep 35: 2583-2591, 2016.

7. Rawlings-Goss RA, Campbell MC and Tishkoff SA: Global population-specific variation in miRNA associated with cancer risk and clinical biomarkers. BMC Med Genomics 7: 53, 2014.

8. Cao WL, Wei WY, Zhan ZX, Xie DY, Xie YB and Xiao Q: The role of miR-647 in human gastric cancer suppression. Oncol Rep 37: 1401-1411, 2017.

9. Kim YW, Kim EY, Jeon D, Liu JL, Kim HS, Choi JW and Ahn WS: Differential microRNA expression signatures and cell type-specific association with Taxol resistance in ovarian cancer cells. Drug Des Devel Ther 8: 293-314, 2014.

10. Livak KJ and Schmittgen TD: Analysis of relative gene expression data using real-time quantitative PCR and the 2(-Delta Delta C(T)) Method. Methods 25: 402-408, 2001.

11. An Y, Zhang Z, Shang Y, Jiang X, Dong J, Yu P, Nie Y and Zhao Q: miR-23b-3p regulates the chemoresistance of gastric cancer cells by targeting ATG12 and HMGB2. Cell Death Dis 6: e1766, 2015.

12. Gisel A, Valvano M, El Idrissi IG, Nardulli P, Azzariti A, Carrieri A, Contino $\mathrm{M}$ and Colabufo NA: miRNAs for the detection of multidrug resistance: Overview and perspectives. Molecules 19: 5611-5623, 2014.

13. Pérez-Tomás R: Multidrug resistance: Retrospect and prospects in anti-cancer drug treatment. Curr Med Chem 13: 1859-1876, 2006

14. Nishida T, Egashira Y, Akutagawa H, Fujii M, Uchiyama K, Shibayama Y and Hirose Y: Predictors of lymph node metastasis in T1 colorectal carcinoma: An immunophenotypic analysis of 265 patients. Dis Colon Rectum 57: 905-915, 2014.

15. Díaz-López A, Moreno-Bueno G and Cano A: Role of microRNA in epithelial to mesenchymal transition and metastasis and clinical perspectives. Cancer Manag Res 6 : 205-216, 2014

16. Sui H, Cai GX, Pan SF, Deng WL, Wang YW, Chen ZS, Cai SJ, Zhu HR and Li Q: miR200c attenuates P-gp-mediated MDR and metastasis by targeting JNK2/c-Jun signaling pathway in colorectal cancer. Mol Cancer Ther 13: 3137-3151, 2014.

17. Lambert $S$ and Bennett V: Postmitotic expression of ankyrinR and beta R-spectrin in discrete neuronal populations of the rat brain. J Neurosci 13: 3725-3735, 1993.

18. De Matteis MA and Morrow JS: The role of ankyrin and spectrin in membrane transport and domain formation. Curr Opin Cell Biol 10: 542-549, 1998.

19. Zhu D and Bourguignon LY: Interaction between CD44 and the repeat domain of ankyrin promotes hyaluronic acid-mediated ovarian tumor cell migration. J Cell Physiol 183: 182-195, 2000.

20. Bourguignon LY, Zhu H, Shao L and Chen YW: Ankyrin-Tiam1 interaction promotes Racl signaling and metastatic breast tumor cell invasion and migration. J Cell Biol 150: 177-191, 2000.

21. Zhu D and Bourguignon LY: The ankyrin-binding domain of CD44s is involved in regulating hyaluronic acid-mediated functions and prostate tumor cell transformation. Cell Motil Cytoskeleton 39: 209-222, 1998.
22. Chen Y, Löhr M and Jesnowski R: Inhibition of ankyrin-B expression reduces growth and invasion of human pancreatic ductal adenocarcinoma. Pancreatology 10: 586-596, 2010.

23. Savas S, Azorsa DO, Jarjanazi H, Ibrahim-Zada I, Gonzales IM, Arora S, Henderson MC, Choi YH, Briollais L, Ozcelik H, et al: NCI60 cancer cell line panel data and RNAi analysis help identify EAF2 as a modulator of simvastatin and lovastatin response in HCT-116 cells. PLoS One 6: e18306, 2011.

24. Lokeshwar VB, Fregien N and Bourguignon LY: Ankyrin-binding domain of CD44(GP85) is required for the expression of hyaluronic acid-mediated adhesion function. J Cell Biol 126: 1099-1109, 1994

25. Jiang W, Zhang Y, Kane KT, Collins MA, Simeone DM, di Magliano MP and Nguyen KT: CD44 regulates pancreatic cancer invasion through MT1-MMP. Mol Cancer Res 13: 9-15, 2015.

26. Miletti-González KE, Chen S, Muthukumaran N, Saglimbeni GN, Wu X, Yang J, Apolito K, Shih WJ, Hait WN and Rodríguez-Rodríguez L: The CD44 receptor interacts with P-glycoprotein to promote cell migration and invasion in cancer. Cancer Res 65: 6660-6667, 2005.

27. Yang X, Iyer AK, Singh A, Choy E, Hornicek FJ, Amiji MM and Duan Z: MDR1 siRNA loaded hyaluronic acid-based CD44 targeted nanoparticle systems circumvent paclitaxel resistance in ovarian cancer. Sci Rep 5: 8509, 2015.

28. Nieh S, Jao SW, Yang CY, Lin YS, Tseng YH, Liu CL, Lee TY, Liu TY, Chu YH and Chen SF: Regulation of tumor progression via the Snail-RKIP signaling pathway by nicotine exposure in head and neck squamous cell carcinoma. Head Neck 37: 1712-1721, 2015.

29. Sein TT, Thant AA, Hiraiwa Y, Amin AR, Sohara Y, Liu Y, Matsuda S, Yamamoto T and Hamaguchi M: A role for FAK in the Concanavalin A-dependent secretion of matrix metalloproteinase-2 and -9. Oncogene 19: 5539-5542, 2000.

30. You T, Gao W, Wei J, Jin X, Zhao Z, Wang C and Li Y: Overexpression of LIMK1 promotes tumor growth and metastasis in gastric cancer. Biomed Pharmacother 69: 96-101, 2015.

31. Wu L, Tanimoto A, Murata Y, Sasaguri T, Fan J, Sasaguri Y and Watanabe T: Matrix metalloproteinase-12 gene expression in human vascular smooth muscle cells. Genes Cells 8: 225-234, 2003.

32. Xie S, Issa R, Sukkar MB, Oltmanns U, Bhavsar PK, Papi A, Caramori G, Adcock I and Chung KF: Induction and regulation of matrix metalloproteinase-12 in human airway smooth muscle cells. Respir Res 6: 148, 2005.

33. Wang B, Xu YF, He BS, Pan YQ, Zhang LR, Zhu C, Qu LL and Wang SK: RNAi-mediated silencing of CD147 inhibits tumor cell proliferation, invasion and increases chemosensitivity to cisplatin in SGC7901 cells in vitro. J Exp Clin Cancer Res 29: 61,2010.

34. Lv FZ, Wang JL, Wu Y, Chen HF and Shen XY: Knockdown of MMP12 inhibits the growth and invasion of lung adenocarcinoma cells. Int J Immunopathol Pharmacol 28: 77-84, 2015.

35. O'Shaughnessy J: Extending survival with chemotherapy in metastatic breast cancer. Oncologist 10 (Suppl 3): 20-29, 2005.

This work is licensed under a Creative Commons Attribution-NonCommercial-NoDerivatives 4.0 International (CC BY-NC-ND 4.0) License. 